\title{
Measuring the Titchener circles and Delboeuf illusions with the method of adjustment
}

\author{
ALEXANDER W. PRESSEY \\ University of Manitoba, Winnipeg, Manitoba, Canada R3T 2N2
}

\begin{abstract}
Two experiments were conducted, one utilizing the Titchener circles and the second the Delboeuf target, to determine the effect of size of contextual circle on degree of distortion. The method of adjustment was used in both experiments. The results were very similar to those obtained by indirect psychophysical techniques. The test-retest reliability coefficient for a composite Titchener circles illusion was +.76 ; for a composite Delboeuf illusion, it was +.77 . The correlation between the two types of illusions was +.30 . It was also found that in both configurations the illusion of expansion was greater than the illusion of shrinkage, which is consistent with the trend in the Mueller-Lyer illusion.
\end{abstract}

The Titchener circles and the Delboeuf illusions, shown in Figure 1, are geometric illusions which have not been empirically investigated to any great extent. Undoubtedly, one reason for the limited research is that, in each case, the standard stimulus is a circle-a configuration which is difficult to manipulate. Thus, most investigators have chosen indirect psychophysical methods such as the method of constant stimuli (Obonai, 1954), the staircase method (Cooper \& Weintraub, 1970), and the method of paired comparisons (Keats, 1964; Zigler, 1960). There are, however, several difficulties with such indirect methods. First, they require a large number of trials to establish the point of subjective equality, with the result that boredom and carelessness may come to play a major role. A second difficulty is that a discrete response such as "longer" or "shorter" is required and one cannot be certain that the subject is responding to the standard circle per se. That is, the subject may be responding to the total size of the target, thus confounding the data with response bias. The problem of insuring that the subject is actually judging what he is asked to judge becomes acute when atypical groups such as retardates, psychotic patients, or very young children are involved. For these reasons, a direct method of measuring illusions is preferred and the main purpose of this report was to describe a method of adjustment for measuring the Titchener circles and Delboeuf illusions. A secondary aim was to determine how reliable the adjustment method is and how well it produces data that are consistent with those obtained with different psychophysical techniques.

This research was supported by the National Research Council of Canada (AO-177). I am indebted to Mr. Les Bell, who devised the apparatus used in this study.

\section{EXPERIMENT 1}

\section{Method}

Apparatus. The apparatus for producing a circle consisted of an oscilloscope, signal generator, and a 10-turn potentiometer. The circle was developed on the oscilloscope by applying one ac voltage to the vertical plates and a second ac voltage, 90 deg out of phase, to the horizontal plates.

The two voltages, with the desired phase relationship, were derived from a series resistor-capacitor arrangement excited by a signal of approximately $100 \mathrm{~Hz}$ from the signal generator. A 10 turn potentiometer, with a vernier drive, controlled the amplitude of the voltage applied to the network and, hence, gave fine control of the diameter of the circle. An isolating transformer allowed the horizontal and vertical voltages to be developed with reference to a common ground.

The device was calibrated by superimposing a transparent window (with the standard circle inscribed on it) over the face plate of the oscilloscope and adjusting the gain of the signal generator or the oscilloscope amplifiers to reproduce the standard for a given potentiometer setting. The potentiometer setting for the standard circle was 5 , and this unit corresponded to a circle with a diameter of $20 \mathrm{~mm}$. On a descending trial the
A



Figure 1. The Delboeuf (A) and Titchener circles (B) configurations. The inner circle in $A$ appears larger than the control circle, which in turn looks larger than the inner circle in $B$. 
potentiometer setting was 10 , and this produced a circle with a diam of $40 \mathrm{~mm}$. A setting of 0 corresponded to a circle of 0 diam. Thus, there was a linear relationship between the reading of the 10-turn potentiometer and the size of the circle. Measurement of the circle was accurate to within $.04 \mathrm{~mm}$.

The illusory targets were drawn on sheets of white paper which were $196 \times 143 \mathrm{~mm}$. The standard circle, which had a diameter of $20 \mathrm{~mm}$, was centered on this sheet and the contextual stimuli were positioned as required. In the Titchener circles, five contextual stimuli were used in all targets, and the distance from the center of the standard circle to the nearest point on the contextual circle was $20 \mathrm{~mm}$. Fourteen Titchener circles targets were drawn in which the contextual circles were 8 , $10,12,14,16,18,20,22,24,28,32,36,40$, and $44 \mathrm{~mm}$ in diam. It should be noted that the control condition consisted of the standard circle and five contextual circles, all of which were $20 \mathrm{~mm}$ in diam. All cards were laminated to protect them from wear.

Procedure. The apparatus was placed on a wooden stand and the subject sat in front of the stand with his chin in a rest so that the viewing distance was held constant at about $60 \mathrm{~cm}$.

For each subject the targets were randomized and were mounted, one by one, in a slot directly below the screen of the oscilloscope. The subject's task was to adjust the circle on the screen to appear equal to the standard circle.

Testing was carried out in two sessions separated by a minimum of $24 \mathrm{~h}$. In each session, one ascending and one descending trial was given successively on each target. The ascendingdescending order was constant within sessions but alternated between sessions. In addition, this order was counterbalanced between subjects. Each ascending trial began with a circle diameter of $0 \mathrm{~mm}$ (a dot) and each descending trial began with a diam of $40 \mathrm{~mm}$. Before each testing period the size and shape of the comparison circle were calibrated with a template to insure that no prolonged changes in voltage had occurred.

Subjects. The subjects, whose average age was approximately 27 years, were 20 volunteers who were paid for participating. The sample consisted of graduate students, faculty, and support staff. Only those who claimed to have good vision, with or without glasses, were asked to participate.

\section{Results and Discussion}

Scores from the potentiometer were converted into diameters of circle in millimeters. Then scores from the four trials were averaged for each size of contextual circle and were recorded as deviations from the control condition. A plus value indicated an illusion in the expected direction and a negative value indicated a reversed illusion. The results, shown in Figure 2, indicate that both expansion and shrinkage effects were present and that both effects increased as negatively accelerated functions of size of contextual circles. Interestingly, in virtually every analogous condition, the expansion illusion was larger than the contraction illusion.

In order to obtain an estimate of reliability, scores on the two sessions were analyzed separately. For each subject, expansion and contraction illusions were summed across conditions and a product moment correlation coefficient was calculated between the two sessions. The resulting value of $r$ was +.76 , which indicates a fair degree of stability across sessions. However, such high correlation coefficients were not found when shrinkage illusions alone $(+.41)$ or expansion illusions

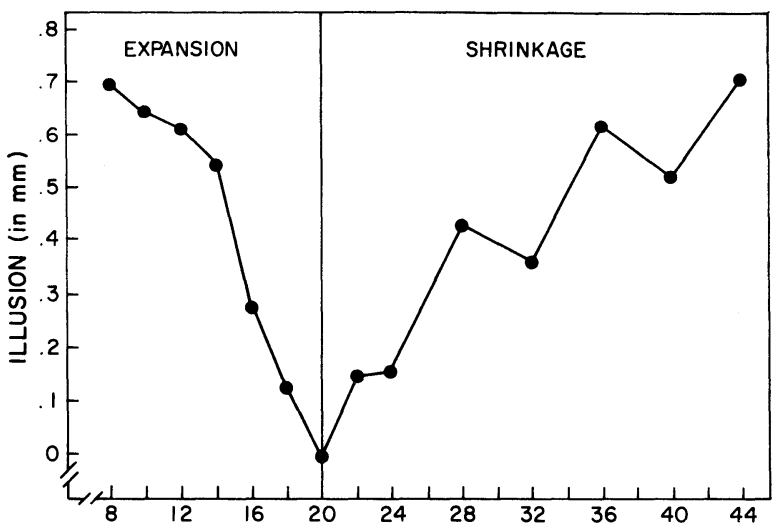

DIAMETER OF CONTEXTUAL CIRCLES (in $\mathrm{mm}$ )

Figure 2. Degree of distortion produced by the Titchener circles target as a function of size of contextual circles.

alone $(+.18)$ were used. This would seem to indicate that, where individual differences are of prime concern, expansion and contraction illusions should be induced and a composite score obtained for each subject.

\section{EXPERIMENT 2}

The purpose of this experiment was to measure the Delboeuf illusion as a function of size of contextual circle by the method of adjustment.

\section{Method}

The apparatus, materials, design, and procedures were very similar to those used in Experiment 1. The diameters of the inducing circles were the same as those used for the Titchener circles. The only difference was that, in this case, the control condition consisted of a single circle. Eighteen of the 20 subjects who participated in the first study were tested on the Delboeuf illusion.

\section{Results and Discussion}

Scores from the potentiometer were converted to diameters of circle in millimeters. Then scores from the four trials were averaged for each condition and were recorded as deviations from the control condition. The results, shown in Figure 3, indicate that the expansion and contraction illusions occurred as expected and that both functions are nonmonotonic. In fact, the patterns for both the shrinkage and the expansion illusion are similar to those reported by the many investigators in Japan (Oyama, 1960), by Piaget, Lambercier, Boesch, and von Albertini (1942), and by Keats (1964). In addition, the overall magnitude of distortion is greater for the expansion illusion than for the shrinkage illusion, a fact that is also supported by virtually every previous study in which the effects of small and large contextual circles were compared.

An attempt was made to assess the test-retest reliability of the Delboeuf illusion and, for this purpose, separate scores for the two sessions were calculated. For 


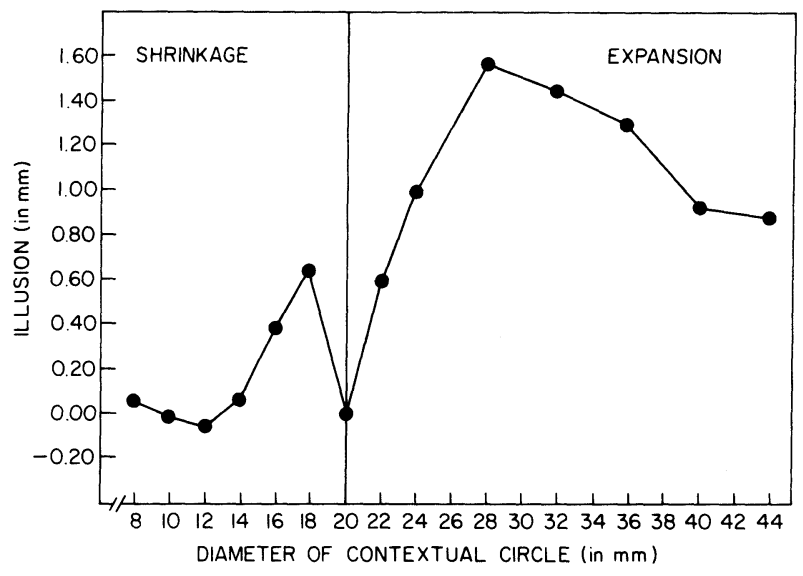

Figure 3. Degree of distortion produced by the Delboeuf target as a function of size of contextual circles.

each subject three scores were obtained. The first was a sum of the expansion illusions; the second was the sum of the shrinkage illusions; the third was a composite of the two illusions. The product moment correlation coefficients were +.69 for the expansion illusion, +.57 for the contraction illusion, and +.77 for the composite score. Thus, the method of adjustment produces a fairly stable Delboeuf illusion and, therefore, can be used to assess individual and group differences in distortion.

In addition, a correlation coefficient of +.30 was found between the Titchener circles and the Delboeuf illusions, which is not significant at the .05 level of confidence.

One of the most interesting results that emerged from these experiments is that the expansion and contraction illusions were not equal. The expansion illusion was greater than the contraction illusion, and this is consistent with the finding that the elongation illusion produced by the outgoing fins is larger than the shrinkage produced by the ingoing fins (e.g., Binet, 1895; Fisher, 1970; Pressey, 1974). The results of this study show that it is not the type of contextual stimuli that are of primary importance but the type of distortion that the standard stimulus undergoes. This suggests the simple hypothesis that distortion occurs in jnd units, and, since the jnd units on the smaller side of a magnitude are smaller than those on the larger side, an equal number of jnds of shrinkage and expansion will produce an unequal amount of illusion as measured on a linear scale. A simple prediction from such an interpretation is that the disparity between the shrinkage and expansion illusions should increase as the absolute size of illusion increases. Such a prediction seems to hold for the Mueller-Lyer illusion (Bayer \& Pressey, 1972; Lewis, 1909) and it appears to hold true for the data shown in Figures 2 and 3.

Finally, it is interesting to note that, if the interpretation for the asymmetry between the shrinkage and expansion illusions given here is correct, then the asymmetry itself is of little value in differentiating among different theories of illusions.

\section{REFERENCES}

Bayer, C. A., \& Pressey, A. W. Geometric illusions as a function of pigmentation of the fundus oculi and target size. Psychonomic Science, 1972, 26, 77-79.

Binet, A. La mesure des illusions visuelles chez les enfants. Revue Philosophique, 1895, 40, 11-25.

Cooper, L. A., \& Weintraub, D. J. Delboeuf-type circle illusions: Interactions among luminance, temporal characteristics, and inducing-figure variations. Journal of Experimental Psychology, 1970, 85, 75-82.

Fisher, G. H. An experimental and theoretical appraisal of the perspective and size-constancy theories of illusions. Quarterly Journal of Experimental Psychology, 1970, 22, 631-652.

KeATS, J. A. An application of the method of paired comparisons to the study of the Delboeuf illusion. Australian Journal of Psychology, 1964, 16, 169-174.

LEWIS, E. O. Confluxion and contrast effects in the Mueller-Lyer illusion. British Journal of Psychology, 1909, 3, 21-41.

OBONAI, T. Induction effects in estimates of extent. Journal of Experimental Psychology, 1954, 47, 57-60.

Oy amA, T. Japanese studies on the so-called geometrical-optical illusions. Psychologia, 1960, 3, 7-20.

Piaget, J., Lambercier, M., Boesch, E., \& von Albertini, B. Introduction a l'etude des perceptions chez l'enfant et analyse d'une illusion relative a la perception visuelle de cercles concentriques (Delboeuf). Archives de Psychologie, 1942, 29 , 1-107.

Pressey, A. W. Evidence for the role of attentive fields in the perception of illusions. Quarterly Journal of Experimental Psychology, 1974, 26, 464-471.

ZigLer, E. Size estimates of circles as a function of size of adjacent circles. Perceptual and Motor Skills, 1960, 11, 47-53.

(Received for publication March 25, 1977.) 